\title{
O Teorema de Kontsevich e os intercâmbios polinomiais
}

\author{
Adailton José da Silva (D)
}

\author{
Amanda Gonçalves Saraiva Ottoni iD
}

José Eloy Ottoni (D

\section{Resumo}

Considere os gráficos de $\mathrm{n}$ polinômios distintos de uma variável real se cruzando em um mesmo ponto. Da esquerda para a direita desse ponto de coincidência, em geral, a ordem desses gráficos muda, os gráficos chegam em uma ordem e saem em outra. Na vizinhança desse ponto, a reordenação desses gráficos sempre pode ser descrita por alguma permutação de $\{1, \cdots, n\}$ ao passarem por ele. Entretanto é válido se questionar se vale a recíproca, isto é; qualquer permutação de $\{1, \cdots, n\}$ descreve uma possível configuração de gráficos de n polinômios que se interceptam em um mesmo ponto (na vizinhança desse ponto)?

Palavras-chave: Teorema de Kontsevich; Intercâmbios Polinomiais; Permutações Separáveis.

\begin{abstract}
Consider the graphs of $\mathrm{n}$ distinct polynomials of a real variable, intersecting at one point. In the neighborhood of this point, it's always possible to describe qualitatively the configuration of these graphs, crossing the point, by some permutation of $\{1, \cdots, n\}$. However, it's compelling to question whether the reciprocal worths, that is to say; does any permutation of $\{1, \cdots, n\}$ describe a possible configuration of $n$ polynomials' graphs intersecting at the same point (in the neighborhood of this point)?
\end{abstract}

Keywords: Kontsevich's Theorem; Polynomial Interchanges; Separable Permutations.

\section{Introdução}

Na matemática é natural associar problemas de contagem ao conjunto das permutações. Por exemplo, questões do tipo: "De quantas maneiras diferentes podemos colorir, com n cores, os vértices de um triângulo?"; "Quantos colares distintos podem ser feitos com n miçangas distintas?"; "De quantas formas podemos somar números inteiros positivos para a soma ser igual a 8?" e muitas outras podem ser tratadas adequadamente com a teoria das permutações e a análise combinatória.

Este trabalho apresenta um outro tipo de problema de contagem, que também será associado ao conjunto de permutações: "Será o número de possíveis intercâmbios polinomiais de n polinômios igual a n! ?", isto é; "O número de configurações possíveis dos gráficos de n polinômios que se intersectam em um ponto, na vizinhança desse ponto, é igual ao número total de permutações de n elementos?". Existirão "permutações proibidas"? A princípio, a resposta parece ser positiva para a primeira pergunta, mas surpreendentemente algo acontece já para o caso $\mathrm{n}=4$, em que o número de intercâmbios polinomiais é menor que 4!. E, de uma maneira ainda mais intrigante; 
como o resultado do número de intercâmbios polinomiais quando $\mathrm{n} \geq 5$ leva em conta diretamente o resultado do caso quando $\mathrm{n}=4$, isto implica numa drástica redução no número de casos possíveis quando $\mathrm{n}$ for grande!

Tal problema foi proposto inicialmente pelo matemático russo Maxim Kontsevich, que apresentou coloquialmente ao seu colega, o matemático francês Étienne Ghys, o resultado que ele obtivera, mostrando que duas configurações são impossíveis de se obter com os gráficos de quatro polinômios intersectando-se em um mesmo ponto. Como conta Ghys em seu livro A singular mathematical promenade [2], Kontsevich, durante uma reunião, entregou-lhe um bilhete de metrô com alguns rabiscos e a palavra "impossible". "Fiquei fascinado: um novo resultado elementar para quatro polinômios em 2009!", descreve Ghys. Mais tarde, Ghys apresentou o problema em um contexto mais geral, em que analisa a situação para mais de quatro polinômios, obtendo um belo e incrivelmente simples resultado descrito em [1] e [2].

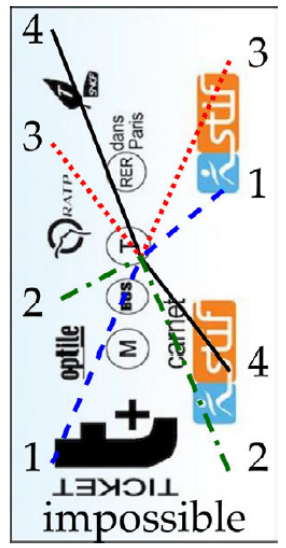

Figura 1: Bilhete de metrô contendo o resultado de Kontsevich [2].

Este artigo foi adaptado da dissertação de mestrado [3], defendida no ano de 2020 e apresentada ao Mestrado Profissional em Matemática em Rede Nacional - Profmat. É importante destacar que a dissertação [3] faz uma transposição do tema, apresentado originalmente em [1], para um contexto de menor complexidade, apresentando a teoria de forma mais detalhada e com mais exemplos ilustrativos, possibilitando, dessa forma, uma menor quantidade de conhecimento preliminar para sua leitura e compreensão.

O artigo é dividido em seis seções e está estruturado da seguinte maneira: A seção 2 expõe as poucas definições preliminares necessárias para a compreensão do trabalho. A seção 3 apresenta o tema central desse trabalho e está dividida em quatro subseções: As subseções 3.1 e 3.2 tratam de casos bem simples no contexto das configurações de gráficos polinomiais; a subseção 3.3 exibe e demonstra o teorema de Kontsevich; na subseção 3.4 é feita a generalização do problema apresentado na seções anteriores. A seção 4 exibe um algoritmo que caracteriza as configurações de gráficos polinomiais. A seção 5 contém as conclusões deste trabalho, que é finalizado com a seção 5 de agradecimentos.

\section{Preliminares}


Pouquíssimos pré-requisitos são necessários para a compreensão dos resultados descritos neste artigo. Basta um pouco de teoria sobre polinômios em $\mathbb{R}[\mathrm{x}]$ e algumas definições e notações sobre permutações de um número finito de elementos. Qualquer polinômio citado neste trabalho deve ser considerado em $\mathbb{R}[\mathrm{x}]$.

Definição 1. O polinômio $P(x)=a_{0}+a_{1} x+a_{2} x^{2}+\cdots+a_{n} x^{n} \in \mathbb{R}[x]$ será dito homogêneo se $P(0)=0$.

Note que $\mathrm{P}(0)=0$ se, e somente se, o seu termo independente $\mathrm{a}_{0}$ é igual a 0 . O gráfico de qualquer polinômio homogêneo passa pela origem.

Definição 2. Seja $P(x)=a_{0}+a_{1} x+a_{2} x^{2}+\cdots+a_{n} x^{n}$ um polinômio em $\mathbb{R}[x]$. A valuação $v(P)$ de $\mathrm{P}(\mathrm{x})$ é o menor número inteiro $\mathrm{k}$ tal que $\mathrm{a}_{\mathrm{k}} \neq 0$. Por convenção, a valuação do polinômio zero é $\infty$ $[2]$.

Seja $\mathrm{P}(\mathrm{x})=\mathrm{a}_{\mathrm{k}} \mathrm{x}^{\mathrm{k}}+\mathrm{a}_{\mathrm{k}+1} \mathrm{x}^{\mathrm{k}+1}+\mathrm{a}_{\mathrm{k}+2} \mathrm{x}^{\mathrm{k}+2}+\cdots+\mathrm{a}_{\mathrm{n}} \mathrm{x}^{\mathrm{n}}$ um polinômio homogêneo com valuação $\mathrm{k}=$ $\mathrm{v}(\mathrm{P}) \neq 0$. Em uma vizinhança da origem, $\mathrm{P}(\mathrm{x}) \approx \mathrm{a}_{\mathrm{k}} \mathrm{x}^{\mathrm{k}}$. Dessa forma é possível concluir que $\mathrm{o}$ gráfico de $\mathrm{P}(\mathrm{x})$ cruza o eixo $\mathrm{x}$ em $(0,0)$ se, e somente se, a valuação $\mathrm{k}=\mathrm{v}(\mathrm{P})$ é um inteiro ímpar.

Além disso, ao considerar dois polinômios distintos $\mathrm{P}_{1}(\mathrm{x})$ e $\mathrm{P}_{2}(\mathrm{x})$, os gráficos de $\mathrm{P}_{1}(\mathrm{x})$ e $\mathrm{P}_{2}(\mathrm{x})$ cruzam-se na origem (isto é, o sinal de $\mathrm{P}_{1}(\mathrm{x})-\mathrm{P}_{2}(\mathrm{x})$ muda em 0 ) se, e somente se, $\mathrm{v}\left(\mathrm{P}_{1}-\mathrm{P}_{2}\right)$ for ímpar.

Definição 3. Considere $[\mathrm{n}]=\{1,2, \cdots, \mathrm{n}\}$. Uma permutação $\sigma$ do conjunto [n] é uma função bijetiva de $[n]$ em $[n]$. Denota-se por $S_{n}$ o conjunto de todas as permutações do conjunto [n] [4].

Note que $\# \mathrm{~S}_{\mathrm{n}}=\mathrm{n}$ !. Uma permutação $\sigma \in \mathrm{S}_{\mathrm{n}}$ será denotada por meio da seguinte notação: $\sigma=(\sigma(1), \sigma(2), \cdots, \sigma(\mathrm{n}))$.

\section{Os Intercâmbios Polinomiais}

O objetivo em vista é estudar e conhecer as possíveis configurações dos gráficos de n polinômios homogêneos próximos à origem. Mais claramente, deseja-se saber quais são as possibilidades de se obter $\mathrm{n}$ polinômios homogêneos $\mathrm{P}_{1}(\mathrm{x}), \mathrm{P}_{2}(\mathrm{x}), \cdots, \mathrm{P}_{\mathrm{n}}(\mathrm{x})$, tais que para $\mathrm{x}$ pequeno e $\mathrm{x}<0$, então $\mathrm{P}_{1}(\mathrm{x})<\mathrm{P}_{2}(\mathrm{x})<\cdots<\mathrm{P}_{\mathrm{n}}(\mathrm{x})$ e para $\mathrm{x}$ pequeno e $\mathrm{x}>0$, então $\mathrm{P}_{\sigma(1)}(\mathrm{x})<\mathrm{P}_{\sigma(2)}(\mathrm{x})<\cdots<\mathrm{P}_{\sigma(\mathrm{n})}(\mathrm{x})$, em que $\sigma$ é uma permutação de $\mathrm{S}_{\mathrm{n}}$.

Para simplificar a notação, cada configuração de gráficos de n polinômios será representada por meio de uma permutação em $\mathrm{S}_{\mathrm{n}}$. Isto é, dizer que $\sigma=(\sigma(1), \sigma(2), \cdots, \sigma(\mathrm{n}))$ é uma possível configuração de gráficos, quer dizer que existem $\mathrm{n}$ polinômios homogêneos $\mathrm{P}_{1}(\mathrm{x}), \mathrm{P}_{2}(\mathrm{x}), \cdots, \mathrm{P}_{\mathrm{n}}(\mathrm{x})$, tais que se $\mathrm{x}<0, \mathrm{P}_{1}(\mathrm{x})<\mathrm{P}_{2}(\mathrm{x})<\cdots<\mathrm{P}_{\mathrm{n}}(\mathrm{x})$ e se $\mathrm{x}>0, \mathrm{P}_{\sigma(1)}(\mathrm{x})<\mathrm{P}_{\sigma(2)}(\mathrm{x})<\cdots<\mathrm{P}_{\sigma(\mathrm{n})}(\mathrm{x})$, em uma vizinhança da origem.

Para uma melhor compreensão, serão considerados exemplos representativos de polinômios possíveis para os casos particulares $n=2, n=3$ e $n=4$ e, finalmente, será analisado o caso geral $n \geq 5$.

\subsection{Caso $n=2$ - Duas Configurações Possíveis}

O caso de dois polinômios é bem simples. Obviamente existem duas possíveis configurações de gráficos, sendo elas: $(1,2)$ e $(2,1)$. Tais configurações estão ilustradas na figura 2, que apresenta dois pares de polinômios homogêneos cujos gráficos satisfazem cada uma das possíveis configurações. 

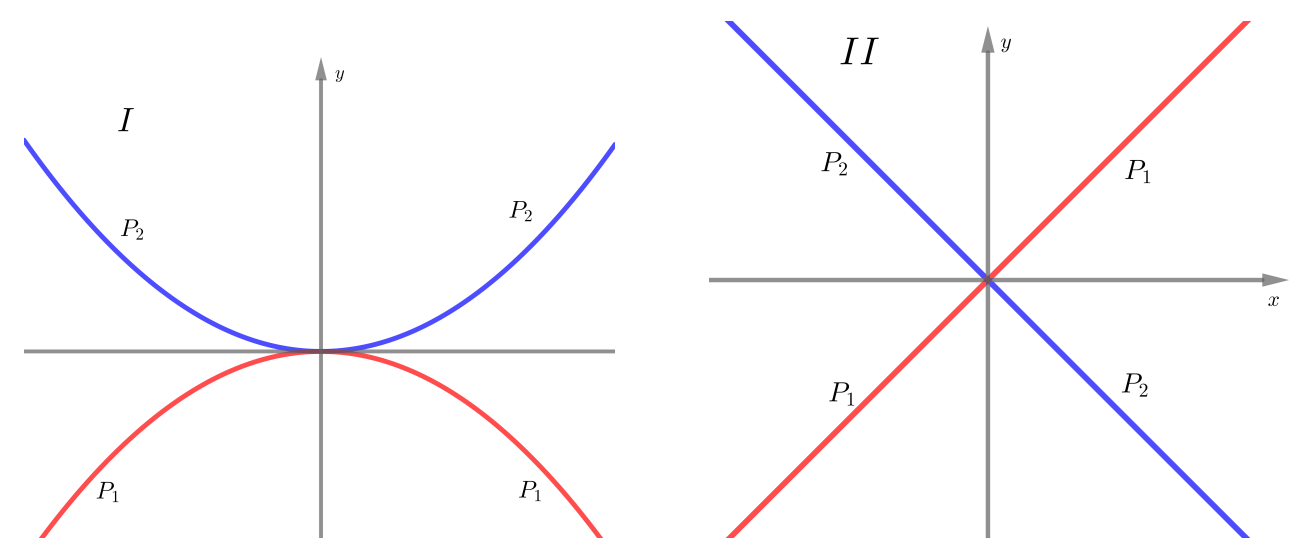

Figura 2: I: $\mathrm{P}_{1}(\mathrm{x})=-\mathrm{x}^{2}$ e $\mathrm{P}_{2}(\mathrm{x})=\mathrm{x}^{2}$. II: $\mathrm{P}_{1}(\mathrm{x})=\mathrm{x}$ e $\mathrm{P}_{2}(\mathrm{x})=-\mathrm{x}$

Esta e as demais figuras apresentadas neste trabalho foram feitas por meio do software livre Geogebra [5].

Neste exemplo, a configuração I é representada pela permutação $(1,2)$, pois, perto da origem, $\mathrm{P}_{1}(\mathrm{x})<\mathrm{P}_{2}(\mathrm{x})$ se $\mathrm{x}<0$ e $\mathrm{P}_{1}(\mathrm{x})<\mathrm{P}_{2}(\mathrm{x})$ se $\mathrm{x}>0$. Analogamente, a configuração II é $(2,1)$, já que $\mathrm{P}_{1}(\mathrm{x})<\mathrm{P}_{2}(\mathrm{x})$ se $\mathrm{x}<0$ e $\mathrm{P}_{2}(\mathrm{x})<\mathrm{P}_{1}(\mathrm{x})$ se $\mathrm{x}>0$ na vizinhança da origem.

\subsection{Caso $n=3$.Seis Configurações Possíveis}

A mesma análise pode ser feita para três polinômios. Novamente, espera-se que existam $\# \mathrm{~S}_{3}=3$ ! possíveis configurações de gráficos, o que de fato acontece, como mostra a figura 3. A figura 3 exibe os gráficos de seis trios de polinômios homogêneos, polinômios esses descritos na tabela 1 . Cada configuração de gráficos mostrada na figura 3 satisfaz uma das configurações em $\mathrm{S}_{3}$.

\begin{tabular}{|c|c|c|c|}
\hline Configuração & $\mathrm{P}_{1}(\mathrm{x})$ & $\mathrm{P}_{2}(\mathrm{x})$ & $\mathrm{P}_{3}(\mathrm{x})$ \\
\hline \hline $\mathrm{I}-(1,2,3)$ & $-\mathrm{x}^{2}$ & 0 & $\mathrm{x}^{2}$ \\
\hline $\mathrm{II}-(1,3,2)$ & $-\mathrm{x}^{2}$ & $\mathrm{x}^{3}$ & 0 \\
\hline $\mathrm{III}-(2,1,3)$ & $\mathrm{x}^{3}$ & 0 & $\mathrm{x}^{2}+\mathrm{x}^{3}$ \\
\hline $\mathrm{IV}-(2,3,1)$ & 0 & $-\mathrm{x}$ & $-\mathrm{x}+\mathrm{x}^{2}$ \\
\hline $\mathrm{V}-(3,1,2)$ & $\mathrm{x}^{3}$ & $\mathrm{x}^{3}+\mathrm{x}^{4}$ & 0 \\
\hline VI - $(3,2,1)$ & $\mathrm{x}^{3}$ & 0 & $-\mathrm{x}^{3}$ \\
\hline
\end{tabular}

Tabela 1: Conjuntos de três polinômios homogêneos, cujos gráficos apresentam as 6 permutações de $\mathrm{S}_{3}$. 

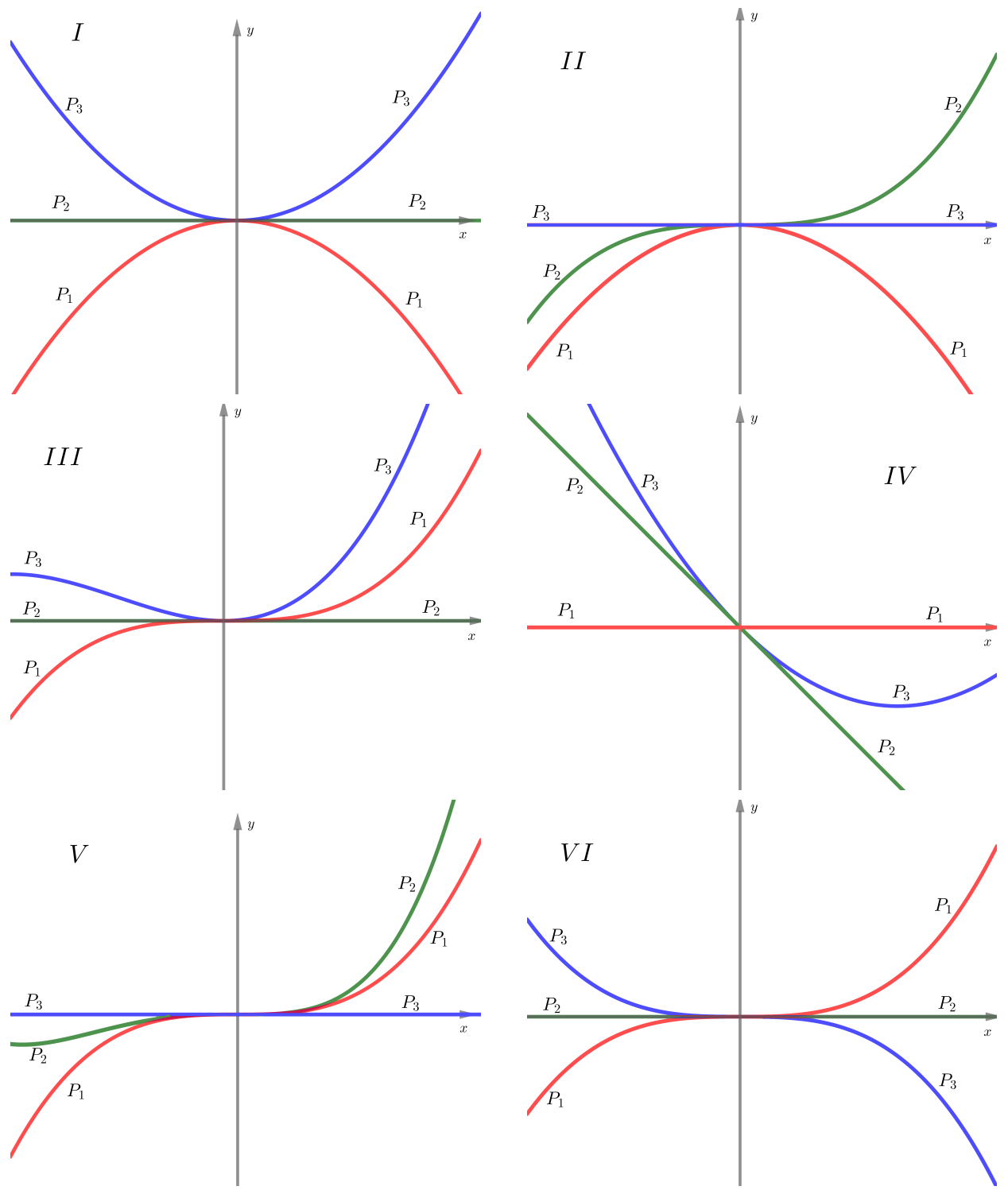

Figura 3: Gráficos dos conjuntos de polinômios descritos na tabela 1.

\subsection{Caso $n=4$. O Teorema de Kontsevich e as Configurações Proibidas}

Para o caso de quatro polinômios algo diferente ocorre. Em princípio, assim como nos casos $\mathrm{n}=2$ e $\mathrm{n}=3$, era esperada a existência de $\# \mathrm{~S}_{4}=4$ ! possíveis configurações de gráficos, entretanto, duas dessas configurações, $(2,4,1,3)$ e $(3,1,4,2)$, são impossíveis de se obter. Esse resultado é o tema central deste trabalho e está enunciado no Teorema 1, descrito abaixo.

Afim de mostrar a existência das demais 22 configurações, a figura 4 exibe os gráficos de 22 quartetos de polinômios homogêneos, todos eles descritos na tabela 2. Cada configuração mostrada 
na figura 4 corresponde a uma permutação permitida, isto é, uma das permutações de $\mathrm{S}_{4}$, exceto $(2,4,1,3)$ e $(3,1,4,2)$.

\begin{tabular}{|c|c|c|c|c|}
\hline Configuração & $\mathrm{P}_{1}(\mathrm{x})$ & $\mathrm{P}_{2}(\mathrm{x})$ & $\mathrm{P}_{3}(\mathrm{x})$ & $\mathrm{P}_{4}(\mathrm{x})$ \\
\hline$\overline{\mathrm{I}}$ & $-\mathrm{x}^{2}$ & $-\mathrm{x}^{4}$ & $\mathrm{x}^{4}$ & $\mathrm{x}^{2}$ \\
\hline II & $-x^{2}$ & 0 & $x^{2}$ & $x^{2}-x^{3}$ \\
\hline III & $-\mathrm{x}^{2}$ & 0 & $-\mathrm{x}^{3}$ & $\mathrm{x}^{2}$ \\
\hline IV & $-\mathrm{x}^{2}$ & 0 & $2 \mathrm{x}^{3}$ & $\mathrm{x}^{4}$ \\
\hline$\overline{\mathrm{V}}$ & $-\mathrm{x}^{2}$ & $\mathrm{x}^{3}$ & $x^{3}+x^{4}$ & $-\mathrm{x}^{3}$ \\
\hline$\overline{\mathrm{VI}}$ & $-\mathrm{x}^{2}$ & $\mathrm{x}^{3}$ & 0 & $-x^{3}$ \\
\hline VII & $-x^{2}+x^{3}$ & $-x^{2}$ & 0 & $x^{2}$ \\
\hline VIII & $-x^{2}$ & $-x^{2}-x^{3}$ & $x^{3}+x^{4}$ & $-x^{3}$ \\
\hline IX & $x^{3}+3 x^{5}$ & $-2 x^{4}$ & 0 & $2 \mathrm{x}^{2}$ \\
\hline $\mathrm{X}$ & $x^{3}-x^{5}$ & $x^{3}+x^{4}$ & $-x^{5}$ & $-x^{3}$ \\
\hline $\mathrm{XI}$ & $\mathrm{x}$ & $-x^{2}$ & 0 & $x^{2}$ \\
\hline XII & $x+x^{2}$ & $-x^{2}-x^{3}$ & $x^{3}+x^{4}$ & $-x^{3}$ \\
\hline XIII & $-2 \mathrm{x}^{4}$ & $x^{5}+x^{6}$ & $-3 x^{3}$ & $2 \mathrm{x}^{2}$ \\
\hline XIV & $\mathrm{x}$ & $2 \mathrm{x}^{3}$ & $-2 \mathrm{x}^{3}$ & $-\mathrm{x}$ \\
\hline $\mathrm{XV}$ & $\mathrm{x}^{3}$ & 0 & $-x^{3}$ & $\mathrm{x}^{2}$ \\
\hline XVI & $\mathrm{x}$ & $\mathrm{x}^{3}$ & 0 & $\mathrm{x}^{2}$ \\
\hline XVII & $2 x^{3}-2 x^{4}$ & $2 x^{3}+2 x^{5}$ & $-\mathrm{x}^{4}$ & $\mathrm{x}^{4}$ \\
\hline XVIII & $2 \mathrm{x}^{3}$ & $\mathrm{x}^{3}$ & 0 & $\mathrm{x}^{4}$ \\
\hline XIX & $-x^{2}$ & 0 & $x^{2}$ & $-\mathrm{x}$ \\
\hline $\mathrm{XX}$ & $-x^{2}$ & $\mathrm{x}^{3}$ & $\mathrm{x}^{4}$ & $-\mathrm{x}$ \\
\hline XXI & $\mathrm{x}^{3}$ & 0 & $x^{2}$ & $-\mathrm{x}$ \\
\hline XXII & $\mathrm{x}$ & $-x^{2}$ & $x^{2}$ & $-\mathrm{X}$ \\
\hline
\end{tabular}

Tabela 2: Exemplos de conjuntos com quatro polinômios homogêneos, cujos gráficos apresentam 22 das 24 permutações de $\mathrm{S}_{4}$. 

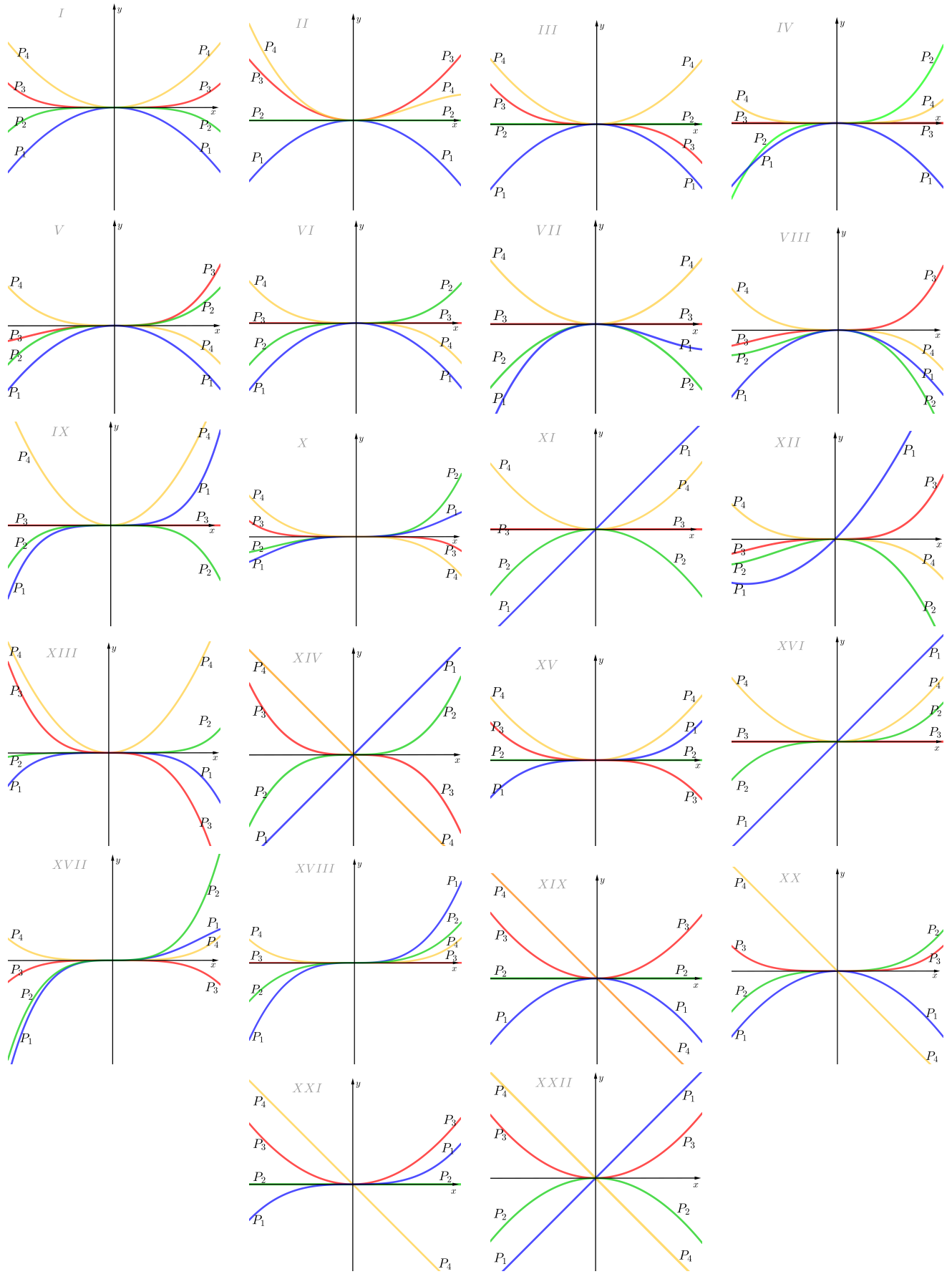

Figura 4: Gráficos dos conjuntos de polinômios descritos na tabela 2.

Teorema 1 (Teorema de Kontsevich [2]). Quatro polinômios homogêneos $\mathrm{P}_{1}(\mathrm{x}), \mathrm{P}_{2}(\mathrm{x}), \mathrm{P}_{3}(\mathrm{x})$, $\mathrm{P}_{4}(\mathrm{x}) \in \mathbb{R}[\mathrm{x}]$ não podem satisfazer: 
I. $\mathrm{P}_{1}(\mathrm{x})<\mathrm{P}_{2}(\mathrm{x})<\mathrm{P}_{3}(\mathrm{x})<\mathrm{P}_{4}(\mathrm{x})$ para $x$ pequeno e $\mathrm{x}<0$,

$\mathrm{P}_{2}(\mathrm{x})<\mathrm{P}_{4}(\mathrm{x})<\mathrm{P}_{1}(\mathrm{x})<\mathrm{P}_{3}(\mathrm{x})$ para $x$ pequeno e $\mathrm{x}>0$, assim como,

II. $\mathrm{P}_{1}(\mathrm{x})<\mathrm{P}_{2}(\mathrm{x})<\mathrm{P}_{3}(\mathrm{x})<\mathrm{P}_{4}(\mathrm{x})$ para $x$ pequeno $e \mathrm{x}<0$,

$\mathrm{P}_{3}(\mathrm{x})<\mathrm{P}_{1}(\mathrm{x})<\mathrm{P}_{4}(\mathrm{x})<\mathrm{P}_{2}(\mathrm{x})$ para $x$ pequeno $e \mathrm{x}>0$.

A figura 5 mostra esquemas de como deveriam se comportar os gráficos de quatro polinômios homogêneos satisfazendo as configurações proibidas de Kontsevich perto da origem. Apenas o item $I$ será demonstrado, pois a demonstração do item $I I$ é completamente análoga (ou também é fácil ver que, se fosse possível obter uma dada configuração de polinômios satisfazendo as condições no item $I$, obteríamos outra obedecendo o item $I I$, e vice-versa, através da simples transformação $\mathrm{x} \rightarrow-\mathrm{x})$.
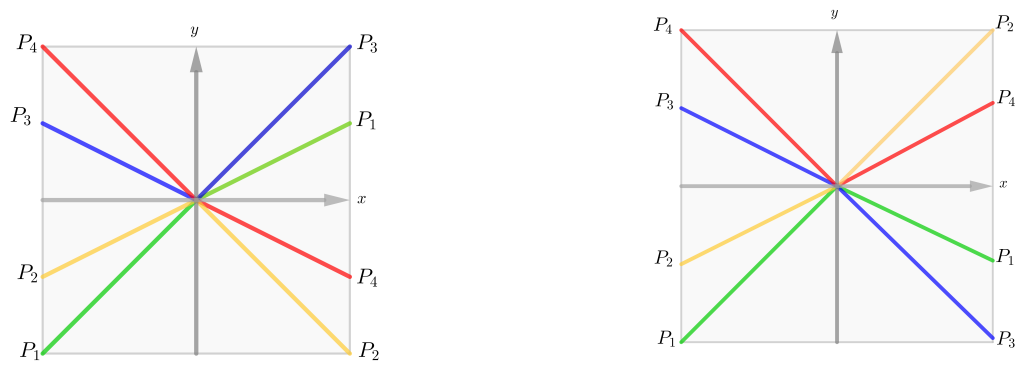

Figura 5: As duas configurações proibidas do Teorema de Kontsevich.

Demonstração. Por contradição, suponha que existam quatro polinômios homogêneos $\mathrm{P}_{1}(\mathrm{x}), \mathrm{P}_{2}(\mathrm{x})$, $\mathrm{P}_{3}(\mathrm{x})$ e $\mathrm{P}_{4}(\mathrm{x})$ satisfazendo as condições expostas em $I$. Substituindo cada $\mathrm{P}_{\mathrm{i}}(\mathrm{x})$ por $\mathrm{P}_{\mathrm{i}}(\mathrm{x})-\mathrm{P}_{1}(\mathrm{x})$ para todo $\mathrm{i}=1, \cdots, 4$, ainda assim $\mathrm{P}_{1}(\mathrm{x}), \mathrm{P}_{2}(\mathrm{x}), \mathrm{P}_{3}(\mathrm{x}), \mathrm{P}_{4}(\mathrm{x})$ são polinômios homogêneos que satisfazem as relações em $I$ e $\mathrm{P}_{1}(\mathrm{x})=0$. A figura 6 apresenta um esquema de como deveriam se comportar os gráficos de $\mathrm{P}_{1}(\mathrm{x}), \mathrm{P}_{2}(\mathrm{x}), \mathrm{P}_{3}(\mathrm{x}), \mathrm{P}_{4}(\mathrm{x})$ perto da origem.

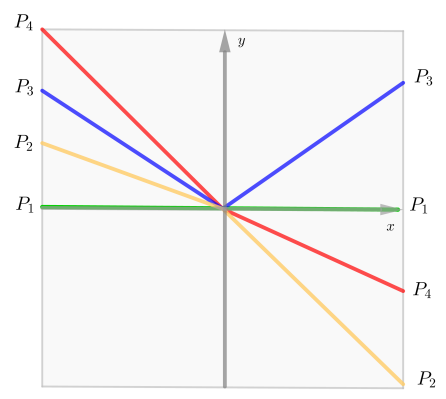

Figura 6: Configuração $(2,4,1,3)$ com $\mathrm{P}_{1}(\mathrm{x})=0$.

Ao analisar a Figura 6, pode-se observar que $\mathrm{P}_{2}(\mathrm{x})$ e $\mathrm{P}_{4}(\mathrm{x})$ mudam de sinal ao passar pela origem, logo as suas valuações $\mathrm{v}\left(\mathrm{P}_{2}\right)$ e $\mathrm{v}\left(\mathrm{P}_{4}\right)$ são ímpares. Além disso, a Figura 6 também mostra que $\mathrm{P}_{3}(\mathrm{x})$ não muda de sinal na origem, o que implica que a sua valuação $\mathrm{v}\left(\mathrm{P}_{3}\right)$ é par. 
Como $0<\mathrm{P}_{2}(\mathrm{x})<\mathrm{P}_{3}(\mathrm{x})<\mathrm{P}_{4}(\mathrm{x})$ para $\mathrm{x}$ negativo pequeno, pode-se concluir que $\mathrm{v}\left(\mathrm{P}_{2}\right) \geq \mathrm{v}\left(\mathrm{P}_{3}\right) \geq$ $\mathrm{v}\left(\mathrm{P}_{4}\right)$ (basta notar que $\mathrm{P}_{\mathrm{i}}(\mathrm{x}) \approx \mathrm{a}_{\mathrm{v}}\left(\mathrm{P}_{\mathrm{i}}\right) \mathrm{x}^{\mathrm{v}\left(\mathrm{P}_{\mathrm{i}}\right)}$ para $\mathrm{x}$ pequeno). Da mesma forma, $\left|\mathrm{P}_{4}(\mathrm{x})\right|<\left|\mathrm{P}_{2}(\mathrm{x})\right|$ para $\mathrm{x}$ pequeno positivo implica que $\mathrm{v}\left(\mathrm{P}_{4}\right) \geq \mathrm{v}\left(\mathrm{P}_{2}\right)$. Isso forçaria as três valuações a serem iguais, mas duas delas são ímpares e uma delas é par. Contradição!

Para quaisquer quatro polinômios homogêneos, o Teorema 1 mostra duas configurações de seus gráficos que são proibidas perto da origem. Será que existem outras configurações proibidas para $\mathrm{n}=4$ ? A tabela 2 juntamente com a figura 4 mostram que não.

As duas configurações descritas no teorema 1 , a saber $(2,4,1,3)$ e $(3,1,4,2)$, serão chamadas de configurações proibidas ou configurações de Kontsevich. Serão chamadas de configurações permitidas aquelas que não são proibidas.

\subsection{Caso $n \geq 5$ - Os Intercâmbios Polinomiais}

$\mathrm{X}_{5}$ Para o caso de $\mathrm{n}=2$ e $\mathrm{n}=3$ polinômios, foi mostrado que todas as $\mathrm{n}$ ! permutações geram configurações possíveis. Para o caso $\mathrm{n}=4$, o teorema de Kontsevich garante que existem duas, das 24 permutações, que são configurações proibidas. Mas o que acontece para $\mathrm{n}$ polinômios quando $\mathrm{n} \geq 5$ ? Esta seção vai apresentar definições e resultados que objetivam responder a esta pergunta.

Definição 4 (Intercâmbio Polinomial). Sejam um número inteiro n $\geq 2$ e $\pi$ alguma permutação em $\mathrm{S}_{\mathrm{n}}$. Dizemos que $\pi$ é um intercâmbio polinomial se existirem $\mathrm{n}$ polinômios $\mathrm{P}_{1}(\mathrm{x}), \ldots, \mathrm{P}_{\mathrm{n}}(\mathrm{x})$ de modo que: $\mathrm{P}_{1}(\mathrm{x})<\mathrm{P}_{2}(\mathrm{x})<\ldots<\mathrm{P}_{\mathrm{n}}(\mathrm{x})$ para $\mathrm{x}$ pequeno e negativo e $\mathrm{P}_{\pi(1)}(\mathrm{x})<\mathrm{P}_{\pi(2)}(\mathrm{x})<\ldots<$ $\mathrm{P}_{\pi(\mathrm{n})}(\mathrm{x})$ para $\mathrm{x}$ pequeno e positivo [1].

Exemplo 1. Todas as permutações de $S_{2}, S_{3}$, bem como as permutações de $S_{4}$ que equivalem às configurações permitidas, são intercâmbios polinomiais.

Definição 5. Sejam $n \geq 5$ um número inteiro e $\pi$ alguma permutação em $S_{n}$. É dito que a permutação $\pi$ contém a permutação $(2,4,1,3)$ se existem inteiros $1 \leq \mathrm{i}_{1}<\mathrm{i}_{2}<\mathrm{i}_{3}<\mathrm{i}_{4} \leq \mathrm{n}$ tais que $\pi\left(\mathrm{i}_{2}\right)<\pi\left(\mathrm{i}_{4}\right)<\pi\left(\mathrm{i}_{1}\right)<\pi\left(\mathrm{i}_{3}\right)$. A definição é análoga se $\pi$ contém a outra permutação proibida $(3,1,4,2)$.

Definição 6 (Permutação Separável). Seja um número inteiro n $\geq 4$ e $\pi$ alguma permutação em $\mathrm{S}_{\mathrm{n}}$. A permutação $\pi$ é dita separável se não contém nenhuma das duas permutações proibidas, [6].

Exemplo 2. A permutação $\sigma=(6,8,3,10,7,5,9,2,1,4) \in \mathrm{S}_{10}$ não é separável uma vez que contém a subpermutação $\sigma=(3,1,4,2)$. De fato, existem quatro índices $\mathrm{i}_{1}=3, \mathrm{i}_{2}=5, \mathrm{i}_{3}=8 \mathrm{e}$ $\mathrm{i}_{4}=10$ tais que: $\mathrm{i}_{1}<\mathrm{i}_{2}<\mathrm{i}_{3}<\mathrm{i}_{4}$ e $\sigma\left(\mathrm{i}_{3}\right)<\sigma\left(\mathrm{i}_{1}\right)<\sigma\left(\mathrm{i}_{4}\right)<\sigma\left(\mathrm{i}_{2}\right)$. Veja a figura 7 . 


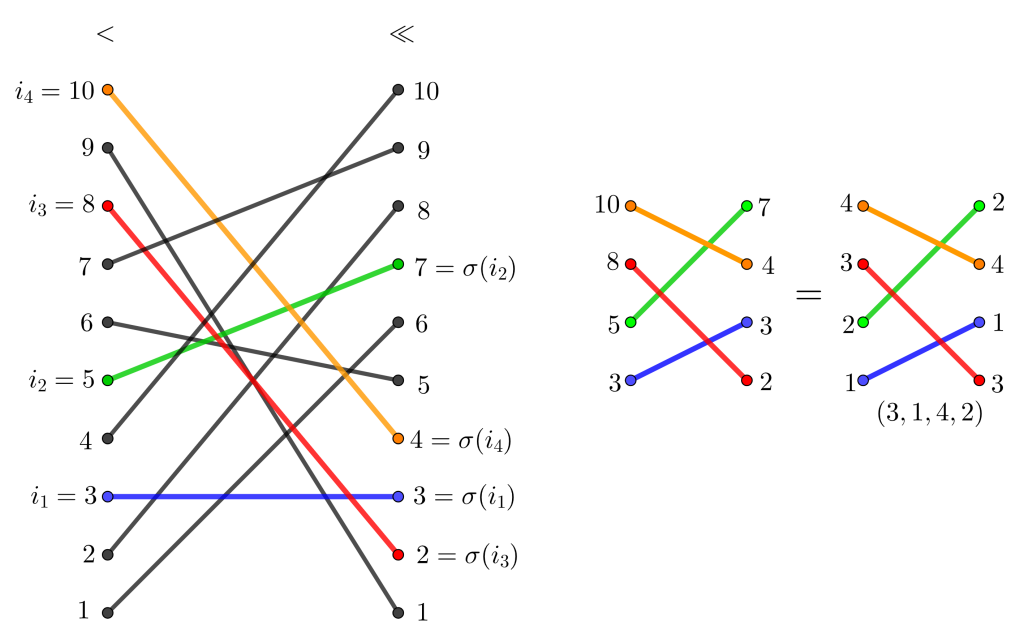

Figura 7: A permutação não separável contém uma das duas permutações proibidas.

No contexto de permutações, matemática discreta e análise combinatória, surge a necessidade de uma definição diferente para intervalo. A definição 7 apresenta uma definição de intervalo em um conjunto discreto que possui uma analogia com a definição de intervalo real.

Definição 7 (Intervalo). Considere o conjunto $[\mathrm{n}]=\{1,2, \cdots, \mathrm{n}\} \quad(\mathrm{n} \geq 2)$, um intervalo de [n] é um subconjunto $J$ contendo uma sequência com $l$ inteiros consecutivos $(2 \leq 1 \leq n)$, isto é, $\mathrm{J}=\{\mathrm{i}, \mathrm{i}+1, \cdots, \mathrm{i}+\mathrm{l}\}$.

Deseja-se caracterizar os intercâmbios polinomiais para um valor qualquer de n (note que isso já foi feito para os casos particulares $n=2, n=3$ e $n=4$ ). Essa caracterização é feita por meio do Teorema 2, descrito ao final desta seção. Para demonstrar o Teorema 2 são necessários os resultados dos Lemas 1 e 2 [2]. É fácil ver que uma condição necessária para que o intercâmbio polinomial exista é que a permutação que o representa seja uma permutação separável! Resta saber se essa condição é suficiente.

Lema 1. Se $\pi$ é uma permutação separável de $\mathrm{S}_{\mathrm{n}}$ para $\mathrm{n} \geq 4$, então existe um intervalo próprio $\mathrm{I}$ de $[\mathrm{n}]$ (I deve ter pelo menos dois elementos e $\mathrm{I} \neq[\mathrm{n}]$ ), cuja imagem $\pi(\mathrm{I})$ também é um intervalo próprio de $[\mathrm{n}]$.

Demonstração. Suponha inicialmente que $\pi(1)<\pi(2)$.

Se $\pi(2)=\pi(1)+1$ o resultado desse lema será obtido pois a imagem de $\{1,2\}$ é o intervalo $\{\pi(1), \pi(2)\}$.

Suponha, então, que $\pi(2)>\pi(1)+1$. Seja $\mathrm{k}$ o menor número inteiro tal que a imagem do intervalo $\mathrm{I}=\{1,2, \ldots, \mathrm{k}\}, \pi(\mathrm{I})$, contenha o intervalo $\mathrm{J}=\{\pi(1), \cdots, \pi(2)\}$ (observe que podem haver elementos de $\pi(\mathrm{I})$ fora de $J)$. Por definição $\pi(1)<\pi(\mathrm{k})<\pi(2)$ pois, caso contrário, $\pi(\{1,2, \cdots, \mathrm{k}-$ 1\}) também conteria $\mathrm{J}$.

Se a imagem $\pi(\mathrm{I})$ for exatamente igual ao intervalo $\mathrm{J}$, o teorema está demonstrado. Caso contrário, escolha um elemento 1 entre 1 e k $(1<\mathrm{l}<\mathrm{k})$, cuja imagem por $\pi$ está fora de J. Veja a figura 8 . 

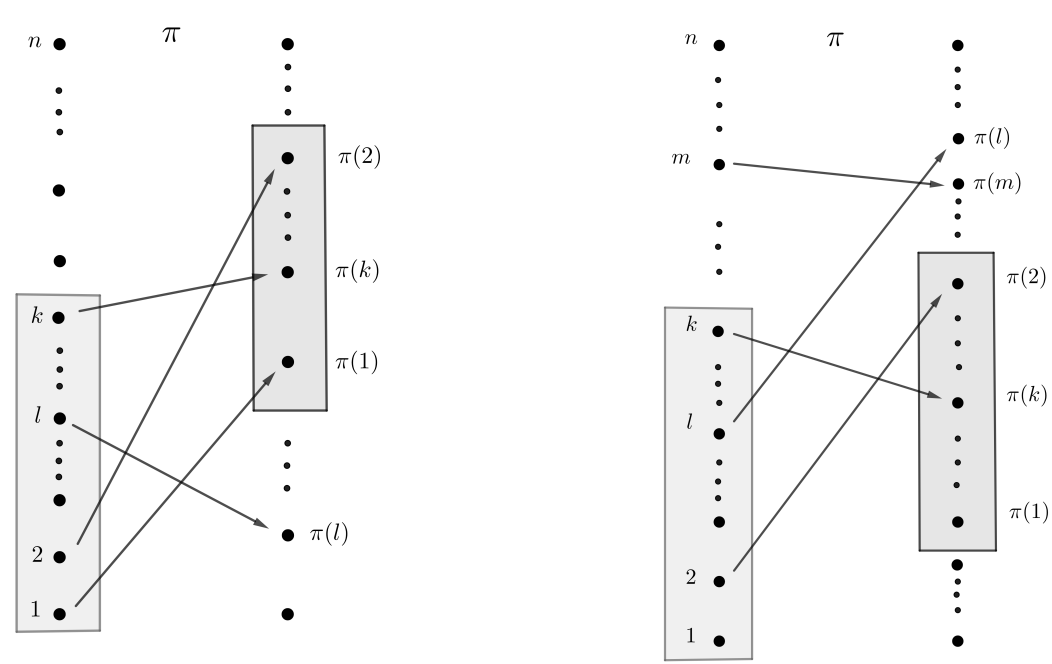

Figura 8: Esquema para facilitar a visualização do resultado do lema 1

Se $\pi(\mathrm{l})<\pi(1)$, os quatro elementos $1<2<\mathrm{l}<\mathrm{k}$ satisfazem $\pi(\mathrm{l})<\pi(1)<\pi(\mathrm{k})<\pi(2)$ e isso quer dizer que $\pi$ contém uma das permutações proibidas. Mas isso é um absurdo, pois $\pi$ é separável. Portanto, todos os elementos de $\pi(\mathrm{I})$ são maiores ou iguais a $\pi(1)$.

Suponha que $\pi(\mathrm{I})$ não seja um intervalo, pois, caso contrário, a demonstração está concluída. Neste caso, há pelo menos uma lacuna em $\pi(\mathrm{I})$, isto é, existe um inteiro $\mathrm{m}$ tal que $2<\mathrm{l}<\mathrm{k}<\mathrm{m}$ e $\pi(\mathrm{k})<\pi(2)<\pi(\mathrm{m})<\pi(\mathrm{l})$, o que gera uma permutação proibida em $\pi$, contradizendo a hipótese.

Se $\pi(1)>\pi(2)$, considere a permutação reversa de $\pi, \bar{\pi}(\mathrm{i})=\mathrm{n}+1-\pi(\mathrm{i})$ (note que para reverter a ordem de um intercâmbio polinomial basta fazer a transformação $\mathrm{x} \rightarrow-\mathrm{x}$, de maneira que $\pi$ é um intercâmbio polinomial se, e somente se, $\bar{\pi}$ também o é). Segue diretamente da definição que $\pi$ contém uma das configurações proibidas se, e somente se, $\bar{\pi}$ contém a outra. Do mesmo modo, dado um intervalo próprio I de $[\mathrm{n}], \pi(\mathrm{I})$ é um intervalo próprio de [n] se, e somente se, $\bar{\pi}(\mathrm{I})$ também o é. Obviamente, $\bar{\pi}(1)<\bar{\pi}(2)$, logo existe um intervalo próprio I de [n], cuja imagem $\bar{\pi}(\mathrm{I})$ também é um intervalo próprio de [n]. O que conclui a demonstração.

O Lema 1 será utilizado como ferramenta para a demonstração do Lema 2. O Lema 2 afirma que para permutações separáveis existe um intervalo de comprimento 2, cuja imagem é um intervalo de comprimento 2. Ele será utilizado para se demonstrar o Teorema 2, que apresenta a caracterização geral para permutações que são intercâmbios polinomiais.

Lema 2. Se $\pi$ é uma permutação separável de $\mathrm{S}_{\mathrm{n}}(\mathrm{n} \geq 4)$, então existem dois números inteiros consecutivos cujas imagens são consecutivas.

Demonstração. Pelo Lema 1, se a permutação $\pi$ é separável, então existe um intervalo próprio $\mathrm{I}_{1}$ de $[\mathrm{n}]$ tal que a imagem $\pi\left(\mathrm{I}_{1}\right)$ também é um intervalo.

Seja $\pi_{1}$ a permutação induzida $\pi$ restrita a $I_{1}$. Obviamente $\pi_{1}$ também é separável e, portanto, pode-se aplicar o Lema 1 na permutação $\pi_{1}$. Logo, existe um intervalo próprio $\mathrm{I}_{2}$ de $\mathrm{I}_{1}$ tal que $\pi\left(\mathrm{I}_{2}\right)$ é um intervalo. 
Ao repetir esse procedimento recursivamente, pode-se garantir a existência de um inteiro k tal que $\mathrm{I}_{\mathrm{k}}$ tem comprimento 2 e $\pi\left(\mathrm{I}_{\mathrm{k}}\right)$ é um intervalo, também de comprimento 2.

Observe que o Lema 2 garante que existe um inteiro a tal que $1 \leq \mathrm{a}<\mathrm{a}+1 \leq \mathrm{n}$ e $\pi(\{\mathrm{a}, \mathrm{a}+1\})$ é um intervalo.

Será enunciado agora o Teorema 2, um dos principais resultados deste trabalho (juntamente com o Teorema 1). Ele faz uma caracterização das permutações que são intercâmbios polinomiais, ou seja, ele poderá responder se uma dada configuração de $n$ polinômios homogêneos, numa vizinhança da origem, é ou não proibida. Até o momento essa resposta é clara apenas se n = 2, 3 ou 4. Além disso, ele mostra que as permutações proibidas são a chave para responder se uma dada configuração de n polinômios é ou não permitida.

Teorema 2 (Teorema de Ghys [2]). Uma permutação de ordem $\mathrm{n} \geq 4$ é um intercâmbio polinomial se, e somente se, for separável.

Demonstração. Seja $\pi$ um intercâmbio polinomial e suponha que $\pi$ não seja separável, o que quer dizer que existem índices $\mathrm{i}_{1}<\mathrm{i}_{2}<\mathrm{i}_{3}<\mathrm{i}_{4}$ tais que $\pi\left(\mathrm{i}_{2}\right)<\pi\left(\mathrm{i}_{4}\right)<\pi\left(\mathrm{i}_{1}\right)<\pi\left(\mathrm{i}_{3}\right)$. Na configuração de polinômios gerada por $\pi$ apague todos os polinômios $\mathrm{P}_{\mathrm{j}}(\mathrm{x})$, em que $\mathrm{j} \neq \mathrm{i}_{\mathrm{k}}$, para $\mathrm{k}=1,2,3,4$. Isso geraria uma configuração proibida, o que é absurdo. A demonstração é análoga para a outra permutação proibida.

Por outro lado, se $\pi$ é uma permutação separável de [n], deseja-se mostrar que existem n polinômios tais que $\mathrm{P}_{1}(\mathrm{x})<\cdots<\mathrm{P}_{\mathrm{n}}(\mathrm{x})$ se $\mathrm{x}<0$ e pequeno, e $\mathrm{P}_{\pi(1)}(\mathrm{x})<\cdots<\mathrm{P}_{\pi(\mathrm{n})}(\mathrm{x})$ se $\mathrm{x}>0$ e pequeno. Para isso será feito indução em $n$.

Se $n=4$ o resultado é válido de acordo com o teorema 1 . Para algum $n \geq 5$, suponha o resultado válido para $n-1$. O Lema 2 garante que existe um inteiro i, $1 \leq \mathrm{i}<\mathrm{n}$, tal que $\{\pi(\mathrm{i}), \pi(\mathrm{i}+1)\}$ é um intervalo. Se $\{\mathrm{i}, \mathrm{i}+1\}$ e $\{\pi(\mathrm{i}), \pi(\mathrm{i}+1)\}$ forem colapsados em um único ponto, será produzida uma permutação $\pi^{\prime}$ de $n-1$ elementos. A permutação $\pi^{\prime}$ é obviamente separável e, portanto, um intercâmbio polinomial por hipótese de indução. Logo existem $n-1$ polinômios afins

$$
\mathrm{P}_{1}(\mathrm{x}), \ldots, \mathrm{P}_{\mathrm{n}-1}(\mathrm{x})
$$

que se interceptam na origem de acordo $\operatorname{com} \pi^{\prime}$.

Inversamente, a partir do i-ésimo polinômio $\mathrm{P}_{\mathrm{i}}(\mathrm{x})$ de $\mathrm{P}_{1}(\mathrm{x}), \ldots, \mathrm{P}_{\mathrm{n}-1}(\mathrm{x})$ pode-se produzir dois outros polinômios $\mathrm{P}_{\mathrm{i}}^{\prime}(\mathrm{x})$ e $\mathrm{P}_{\mathrm{i}}^{\prime \prime}(\mathrm{x})$ a fim de se produzir um conjunto de $\mathrm{n}$ polinômios

$$
\mathrm{P}_{1}(\mathrm{x}), \ldots, \mathrm{P}_{\mathrm{i}-1}(\mathrm{x}), \mathrm{P}_{\mathrm{i}}^{\prime}(\mathrm{x}), \mathrm{P}_{\mathrm{i}}^{\prime \prime}(\mathrm{x}), \mathrm{P}_{\mathrm{i}+1}(\mathrm{x}), \ldots, \mathrm{P}_{\mathrm{n}-1}(\mathrm{x})
$$

que se cruzam de acordo com $\pi$. Para isso, basta definir $\mathrm{P}_{\mathrm{i}}^{\prime}(\mathrm{x})=\mathrm{P}_{\mathrm{i}}(\mathrm{x})$ e $\mathrm{P}_{\mathrm{i}}^{\prime \prime}(\mathrm{x})=\mathrm{P}_{\mathrm{i}}(\mathrm{x})+(-\mathrm{x})^{\mathrm{N}}$ para um valor suficientemente grande de $\mathrm{N}$ tal que $\mathrm{N}>\max \left\{\mathrm{v}\left(\mathrm{P}_{\mathrm{k}}\right), \mathrm{k}=1, \cdots, \mathrm{n}-1\right\}$, onde $\mathrm{v}\left(\mathrm{P}_{\mathrm{k}}\right)$ denota a valuação do polinômio $\mathrm{P}_{\mathrm{k}}(\mathrm{x})$. Note que $\mathrm{N}$ é tomado suficientemente grande de modo que para $\mathrm{x}$ próximo de 0 tenhamos $(-\mathrm{x})^{\mathrm{N}}$ tão próximo de 0 que tanto $\mathrm{P}_{\mathrm{i}}^{\prime}(\mathrm{x})$ quanto $\mathrm{P}_{\mathrm{i}}^{\prime \prime}(\mathrm{x})$ ficam estritamente entre $\mathrm{P}_{\mathrm{i}-1}(\mathrm{x})$ e $\mathrm{P}_{\mathrm{i}+1}(\mathrm{x})$. Além disso, basta tomar $\mathrm{N}$ par quando $\pi(\mathrm{i}+1)>\pi(\mathrm{i})$ e $\mathrm{N}$ ímpar quando $\pi(\mathrm{i}+1)<\pi(\mathrm{i})$. Com isso, conclui-se que $\pi$ é um intercâmbio polinomial, como desejado.

O Lema 2, juntamente com o Teorema 2, fornecem um simples algoritmo para responder se uma dada configuração de $n \geq 4$ polinômios homogêneos é proibida ou permitida. 


\section{Algoritmo para a Caracterização de Intercâmbios Polinomiais:}

Dada uma permutação $\pi \in \mathrm{S}_{\mathrm{n}}(\mathrm{n} \geq 4)$, deseja-se saber se $\pi$ é ou não um intercâmbio polinomial. De acordo com o Lema 2, se $\pi$ é um intercâmbio polinomial então existem dois números inteiros consecutivos cujas imagens por $\pi$ são consecutivas. Baseado nisso e na ideia da demonstração apresentada no Teorema 2, o algoritmo abaixo é capaz de detectar se $\pi$ é ou não uma possível configuração de n polinômios homogêneos.

\section{Algoritmo 1}

(1) $\mathrm{n} \leq 3 ?$

i. Sim $\rightarrow \pi$ é um intercâmbio polinomial.

ii. Não $\rightarrow$ siga para o passo (2).

(2) Procure por dois inteiros consecutivos cujas imagens sejam consecutivas.

(3) Encontrou?

i. Sim $\rightarrow$ Colapse esses dois pontos, gerando uma sequência de $n-1$ elementos e volte ao passo (1).

ii. Não $\rightarrow \pi$ não é um intercâmbio polinomial.

O algoritmo inicia-se com uma permutação de n elementos e, toda vez que a resposta do passo (3) for sim, é subtraído uma unidade no número de elementos da sequência. Ele para quando $\mathrm{n} \leq 3$ ou quando a resposta do passo (3) for não. Para ilustrar seu funcionamento considere os exemplos a seguir.

Exemplo 3. O exemplo 2 mostra que a permutação $\sigma=(6,8,3,10,7,5,9,2,1,4) \in \mathrm{S}_{10}$ não é um intercâmbio polinomial. O algoritmo chega à mesma conclusão em apenas duas rodadas, como mostra a figura 9 .

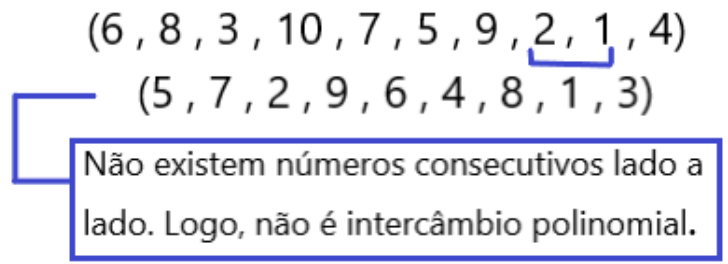

Figura 9: Algoritmo 1 aplicado à permutação do exemplo 2

Na primeira rodada o primeiro par de inteiros consecutivos encontrados é $(2,1)$, que são colapsados e transformados em (1). Subtrai-se uma unidade de todos os elementos maiores que o menor número colapsado, obtendo-se uma permutação em $\mathrm{S}_{9}$. Nessa nova permutação não existe nenhum par de inteiros consecutivos lado a lado, o que permite concluir que $\sigma$ não é um intercâmbio polinomial. 
Exemplo 4. Considere a permutação $\pi=(10,8,9,7,3,5,4,6,2,1) \in \mathrm{S}_{10}$. A figura 10 mostra um esquema representando a aplicação do Algoritmo 1 a essa permutação. Note que com oito rodadas pode-se concluir que $\pi$ é um intercâmbio polinomial.

$$
\begin{gathered}
(10,8,9,7,3,5,4,6,2,1) \\
(9,8,7,3,5,4,6,2,1) \\
(8,7,3,5,4,6,2,1) \\
(7,3,5,4,6,2,1) \\
(6,3,4,5,2,1) \\
(5,3,4,2,1) \\
\left(\frac{3,3,2,1)}{(3,2,1)}\right.
\end{gathered}
$$

Figura 10: Algoritmo 1 aplicado à permutação $\pi$

A dissertação de mestrado [3] contém um exemplo que apresenta dez polinômios cuja configuração de seus gráficos perto da origem é precisamente a permutação $\pi$.

Responder se uma dada configuração de n polinômios homogêneos é ou não permitida parecia ser um processo complicado. Entretanto o Algoritmo descrito acima, de complexidade quadrática, exibe uma forma simples e eficiente de se resolver o problema.

As permutações separáveis são bem conhecidas nas áreas da ciência da computação e combinatória matemática e sabe-se que, assintoticamente, a razão do número de permutações separáveis pelo número total de permutações vai a zero, de onde se depreende que elas são, na verdade, muito raras, quando n é grande [1]. Esse fenômeno pode ser observado na Tabela 3.

A Tabela 3 apresenta a proporção do número de intercâmbios polinomiais, pelo número total de permutações em $\mathrm{S}_{\mathrm{n}}$, quando $\mathrm{n}$ varia de 4 a 12. Para cada valor de $\mathrm{n}$ foram realizadas 300 simulações usando o algoritmo 1, por meio do software Scilab [7]. Em cada simulação foi gerada uma permutação aleatória de $S_{n}$ que era classificada como intercâmbio ou não intercâmbio polinomial. Ao final das 300 simulações calculou-se a proporção do número de intercâmbios encontrados.

\begin{tabular}{|c|c||c|c|}
\hline $\mathrm{n}$ & $\% \mathrm{IPn}$ & $\mathrm{n}$ & $\% \mathrm{IPn}$ \\
\hline 4 & 0.9167 & 9 & 0.1300 \\
5 & 0.7600 & 10 & 0.0667 \\
6 & 0.5900 & 11 & 0.0300 \\
7 & 0.4233 & 12 & 0.0200 \\
8 & 0.2600 & & \\
\hline
\end{tabular}

Tabela 3: \%IPn: Proporção do número de intercâmbios polinomiais pelo número total de permutações em $\mathrm{S}_{\mathrm{n}}$, quando $\mathrm{n}$ varia de 4 a 12 .

O código utilizado para a realização das simulações contidas nesta seção está descrito a seguir: 


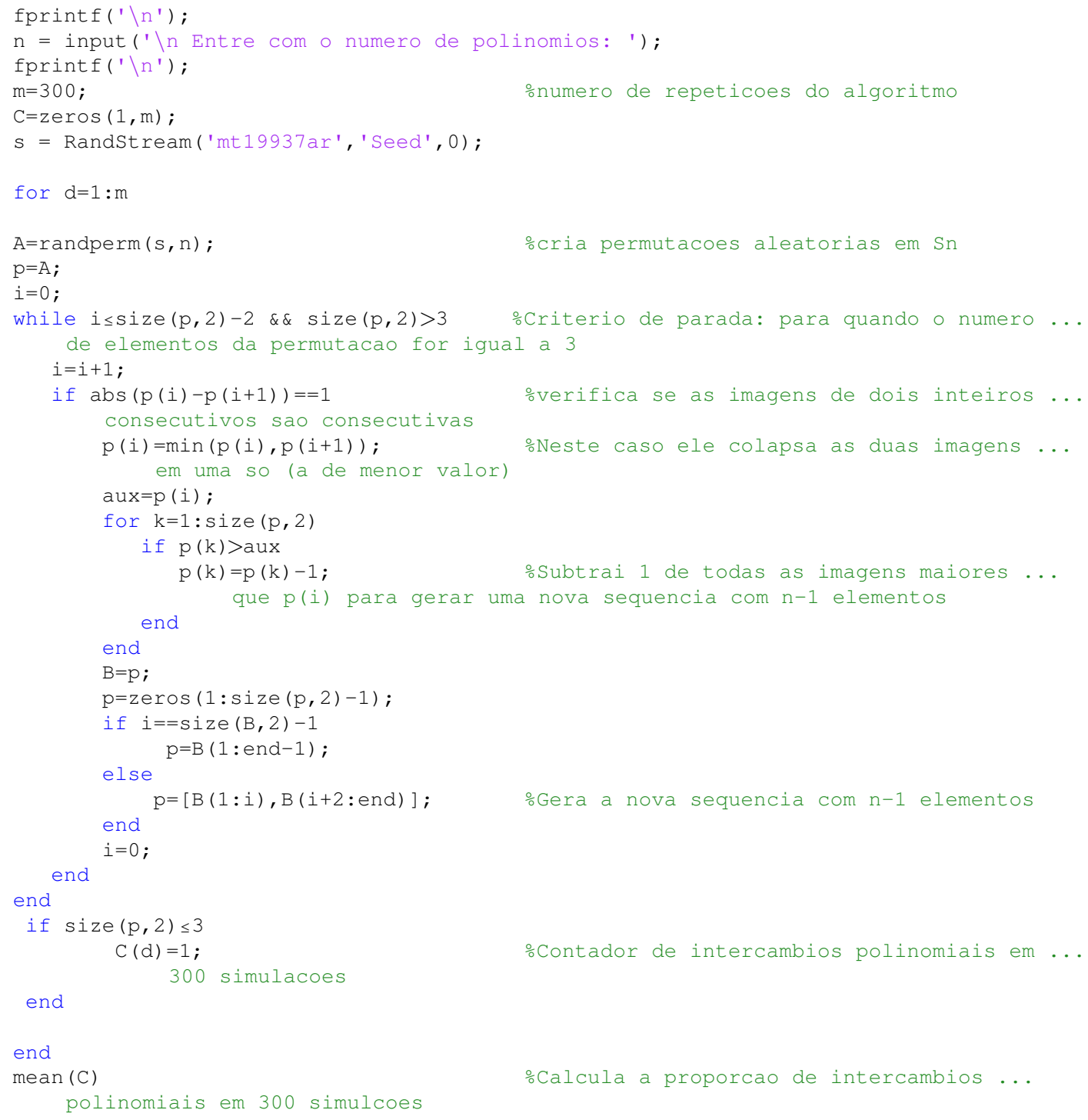

É possível observar [1] que, embora as demonstrações tenham sido feitas para polinômios homogêneos cruzando-se na origem, pode-se generalizar facilmente esse resultado para polinômios não necessariamente homogêneos, interceptando-se em um ponto qualquer, ou até mesmo funções analíticas! Curiosamente, pode-se encontrar exemplos de quatro funções suaves, não analíticas que se interceptam-se, obedecendo às configurações proibidas de Kontsevich!

Mas afinal, o que essas permutações têm de tão especial? Isso, sem dúvida, é um dos extraordinários mistérios da matemática. 


\section{Conclusões}

Este artigo apresentou o Teorema de Kontsevich, que exibe duas configurações impossíveis para os gráficos de quatro polinômios que passam pela origem, em uma vizinhança dessa. As configurações proibidas de Kontsevich são usadas para se caracterizar as configurações impossíveis para os gráficos de cinco ou mais polinômios que passam pela origem, na vizinhança dessa. Um algoritmo simples foi proposto para detectar as configurações proibidas dos gráficos de $n>4$ polinômios que passam pela origem. Por sua simplicidade, esse trabalho pode ser usado como tema extracurricular para alunos do ensino médio. Além disso pode oferecer interessantes desafios para olimpíadas.

\section{Agradecimentos}

Os autores gostariam de agradecer ao revisor anônimo pelas valiosas sugestões.

\section{Referências}

[1] Ghys, É. "Intersecting Curves (Variation on an Observation of Maxim Kontsevich)." The American Mathematical Monthly, vol. 120, n³, 2013, pp. 232-242. JSTOR,

[2] Ghys, É. A singular mathematical promenade. Lyon, ENS Éditions, 2017. Volume 1. ISBN 978-2-84788-940-6.

[3] Silva, A. J. da, Ottoni, A. G. S., Ottoni, J. E. Teorema de Kontsevich e os Intercâmbios Polinomiais. Trabalho de Conclusão de Curso do Mestrado Profissional em Matemática em Rede Nacional - 2020.

[4] Garcia, A., Lequain, Y. Elementos de álgebra. (6 $6^{\mathrm{a}}$ edição). Instituto Nacional de Matemática Pura e Aplicada - Impa, 2018. ISBN: 978-85-244-0450-4.

[5] Geogebra (2020) [Free Software Foundation]. https://www.geogebra.org

[6] Kitaev, S. Patterns in permutations and words. Monographs in Theoretical Computer Science. An EATCS Series, Berlin: Springer-Verlag, 2011, pp. 57-66.

[7] Scilab (versão 6.11) (2021) [Open source software for numerical computation]. https://www.scilab.org/

Adailton José da Silva

Universidade Federal de São João del-Rei <ajsgalan@yahoo.com.br>

Amanda Gonçalves Saraiva Ottoni Universidade Federal de São João del-Rei <amandagso@ufsj.edu.br>

José Eloy Ottoni

Universidade Federal de São João del-Rei $<$ jeottoni@ufsj.edu.br>

Recebido: $12 / 07 / 2021$

Publicado: 03/03/2022 